\title{
THU0243 CAN THE INCREASE OF LOBULES / FOCI RATIO BE HISTOPATHOLOGICAL EVIDENCE OF PRIMARY SJÖGREN'S SYNDROME
}

B. Ünal ${ }^{1}, \underline{\text { V. Yazısız }}{ }^{2}$, G.Ö. Elpek ${ }^{1}$, M. Göçer ${ }^{3}$, M.E. Terzioğlu ${ }^{2} .{ }^{1}$ Pathology; ${ }^{2}$ Rheumatology; ${ }^{3}$ Internal Medicine, Akdeniz University, ANTALYA, Turkey

Background: Minor salivary gland biopsy from the lip region is commonly used for primary Sjögren's syndrome (SS) diagnosis. It is accepted that changes of histopathology is the most important component of diagnostic criteria. In this study, we analyzed the relationship between histopathological changes and laboratory findings/clinical features in SS patients and control group.

Objectives: The aim of this study was to determine the histopathological changes in minor salivary tissue for SS diagnosis

Methods: A total of 69 minor salivary gland biopsies (29 Sjögren's syndrome and 40 controls) were included in the study. Biopsies were evaluated by a blind pathologist unaware of the diagnosis. Histopathological findings were noted as ductal dilatation, fat tissue percentage $(<10 \%$ or $>10 \%)$, acinar atrophy, ductal/ acinar ratio and lobules/foci ratio. It was evaluated whether there was a relationship between histological changes and age, gender, ANA, anti-SSA, anti-SSB, RF and Chisholm scores.

Results: The lobules/foci ratio of patients with Sjögren's syndrome was significantly higher than the control group $(p<0.001)$. The cutoff value of the lobules/foci ratio for SS diagnosis was 0.8 . Furthermore, there was a significant correlations between the lobules/foci ratio greater than 0.8 and antibodies (ANA, RF, anti-Ro) positivity and Chisholm score $(p<0.01)$. Other histopathological findings such as ductal dilatation, fat tissue percentage, acinar atrophy, and ductal/atherine ratio do not distinguish SS from non-SS.

Conclusions: The results of this study revealed that only the lobules/foci ratio from histopathological changes has a strong relation with SS diagnosis. It can be important for pathologists to distinguish SS.

Disclosure of Interest: None declared

DOI: 10.1136/annrheumdis-2017-eular.4014

\section{THU0244 THE ROLE OF STEM CELL-LIKE MEMORY T CELLS IN SYSTEMIC LUPUS ERYTHEMATOSUS}

Y.J. Lee, H. Kwon, E.B. Lee. Seoul National University, Seoul, Korea, Republic Of

Background: The stem cell-like memory $\mathrm{T}$ (Tscm) cell, a special subset of memory $T$ cell, has a potential to self-renew and differentiate into wide spectrum of T cells ${ }^{1}$. However, the role of Tscm in autoimmune disease such as systemic lupus erythematosus (SLE) remains to be unknown.

Objectives: To investigate the levels of Tscm cells in SLE patients compared with healthy controls and to understand the functional characteristics of the Tscm cells from SLE patients.

Methods: Fifty two SLE patients and 57 healthy controls (HCs) were enrolled. To detect Tscm cells which are designated as $\mathrm{CD}^{+} \mathrm{CD}^{+} / \mathrm{CD}^{+}{ }^{+} \mathrm{CD} 45 \mathrm{RO}-\mathrm{CCR}^{+}$ $\mathrm{CD} 2 \mathrm{~L}^{+} \mathrm{CD} 45 \mathrm{RA}^{+} \mathrm{CD} 27^{+} \mathrm{CD} 28^{+} \mathrm{CD} 127^{+} \mathrm{CD} 122^{+} \mathrm{CD}^{2} 5^{\text {hi }}$, flow cytometry was performed after staining peripheral blood mononuclear cells (PBMCs, $1 \times 10^{7}$ cells $/ \mathrm{mL}$ ) with fluorescence-conjugated antibodies. To ascertain the differentiation ability of Tscm cells, different subsets of $T$ cells including naïve-like $T$, central memory $T$, effector memory $T$ and follicular helper $T$ cells (Tfh) were evaluated after stimulating the isolated Tscm cells with anti-CD3/CD28 antibodies for 6 days. To investigate the function of the Tfh cells derived from Tscm cells, secreted IgG antibodies were measured from the supernatants by ELISA after incubating Tfh -containing differentiated Tscm cells with autologous B cells and SEB for 6 days. Results: Among the naive-like $\mathrm{T}\left(\mathrm{CD}^{+} \mathrm{CD4}^{+} \mathrm{CCR}^{+} \mathrm{CD}^{2} 5 \mathrm{RO}^{-} \mathrm{CD} 45 \mathrm{RA}^{+}\right.$ $\mathrm{CD} 2 \mathrm{~L}^{+}$) cells, the proportion of $\mathrm{CD}^{+}$and $\mathrm{CD}^{+} \mathrm{Tscm}$ cells was significantly higher in SLE patients than HCs (for CD4 ${ }^{+} \mathrm{T}$ cells, $2.29 \pm 0.22$ (SLE) vs $1.11 \pm 0.13$

A In CD4+ naive-like T

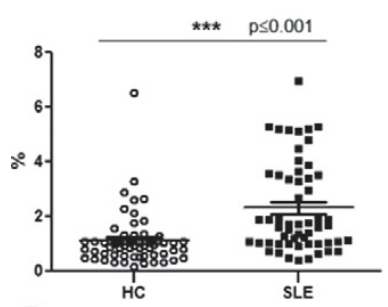

B $\quad \ln \mathrm{CD} 4+\mathrm{T}$

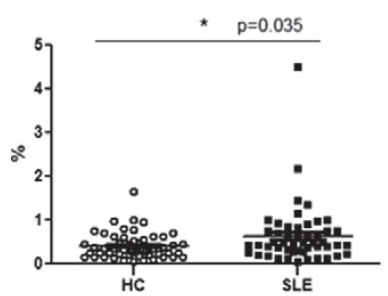

In CD8+ naive-like $T$

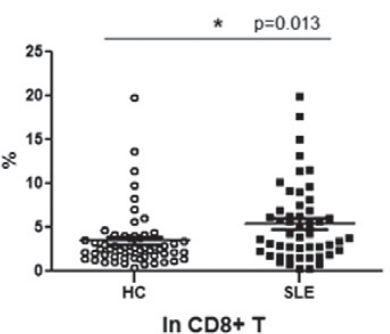

ns

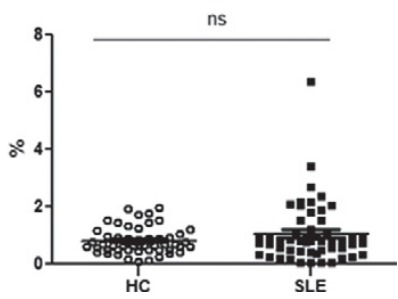

$(\mathrm{HC})$, p-value $\leq 0.001$; for $\mathrm{CD}^{+} \mathrm{T}$ cells, $5.36 \pm 0.63$ (SLE) vs $3.41 \pm 0.44(\mathrm{HC})$, $\mathrm{p}$-value $=0.013)$. Among the total $\mathrm{CD} 4^{+} \mathrm{T}$ cells, the ratio of $\mathrm{Tscm}$ to $\mathrm{CD} 4^{+} \mathrm{T}$ cells was higher in SLE patients than in healthy controls $(0.63 \pm 0.10$ (SLE) vs $0.40 \pm 0.04$ $(\mathrm{HC}$ ), p-value $=0.035$ ) (Fig 1). SLE and $\mathrm{HC}$ Tscm cells could differentiate into naive-like $\mathrm{T}$, central memory $\mathrm{T}$, effector memory $\mathrm{T}$ cells and renewed themselves in response to $\alpha-C D 3 / C D 28$ stimuli. In addition, in response to the same stimuli, SLE Tscm cells could differentiate into more Tfh cells than $\mathrm{HC}$ Tscm cells could (for $\mathrm{CXCR5}^{+} \mathrm{ICOS}^{+} \mathrm{PD}-1^{+}$cells in CD4 $4^{+}$T cells, $9.30 \%$ (SLE) vs $5.41 \%(\mathrm{HC})$ and for $\mathrm{Bcl}^{+} 6^{+}$cells in $\mathrm{CD}^{+}{ }^{+} \mathrm{T}$ cells, $34.29 \%$ (SLE) vs $19.20 \%(\mathrm{HC})$ ). Tscm-derived Tfh cells could help B cells produce antibodies in vitro (12007.81 \pm 4457.54 (SLE) vs $2082.18 \pm 517.82(\mathrm{HC})$ ).

Conclusions: Proportions of Tscm cells among T cells is increased in SLE patients compared with HCs and the Tscm cells may help maintain SLE by its ability to differentiate into Tfh cells.

References:

[1] Luca Gattinoni et al, A human memory T cell subset with stem cell-like properties. Nature Medicine, 2011, 17: 1290-U325.

Disclosure of Interest: None declared

DOI: 10.1136/annrheumdis-2017-eular.2116

\section{THU0245 MAMMALIAN TARGET OF RAPAMYCIN PATHWAY MIGHT CONTRIBUTE THE MINOR SALIVARY GLAND CHANGES IN BOTH SJOGREN'S SYNDROME AND SYSTEMIC SCLEROSIS PATIENTS}

Z.Z. Gumus ${ }^{1}$, F. Cakalagaoglu ${ }^{2}$, Z. Soypacaci ${ }^{3}$, M. Ozmen ${ }^{4}$, D. Solmaz ${ }^{4}$, S. Gucenmez ${ }^{4}$, O. Gercik ${ }^{4}$, S. Akar ${ }^{4} .{ }^{1}$ Internal Medicine; ${ }^{2}$ Pathology;

${ }^{3}$ Nephrology; ${ }^{4}$ Rheumatology, Izmir Katip Celebi University Ataturk Education and Research Hospital, Izmir, Turkey

Background: The Mammalian Target of Rapamycin (mTOR), a multiprotein complex, controls cell growth, proliferation, survival and plays an important role in immunity and inflammation. Transforming Growth Factor- $\beta 1$ (TGF- $\beta 1$ ) leads to an increase in mTOR levels by protein kinase B (PKB or Akt) pathway. Phosphatase and tensin homolog (PTEN) affects mTOR pathway by inhibiting Akt. Recently, increased activities of TGF- $\beta 1$ and mTOR were established in a mouse model of SSc. However the role of mTOR pathway, which might be a potential treatment target, in the human salivary gland inflammation is not evaluated systematically before.

Objectives: Therefore, we aimed to examine the activity of mTOR pathway and TGF- $\beta 1$ in human minor salivary gland biopsies of Sjogren's Syndrome (SjS) and systemic sclerosis (SSc) patients.

Methods: Immunohistochemical technique was used to analyze the expression of mTOR, PTEN and TGF- $\beta 1$ in human minor salivary gland biopsies of SjS, SSc and SjS-SSc overlap patients. Immunohistochemical staining was evaluated by light microscopy and staining intensity was scored semi-quantitatively (Figure 1). The biopsy specimens were also examined for the degree of fibrosis.

Results: Formalin fixed sections of minor salivary gland biopsies of $58 \mathrm{SjS}$ (57 female [98.3\%]), 14 SSc (13 female [92.8\%]) and 23 SjS-SSc overlap patients (all female) were included in the study. All biopsy specimens of SjS and overlap patients had a focus score of $\geq 1$. There is no significant difference regarding mTOR expression between groups ( $P=0.622)$. mTOR expression was found in $94.4 \%$ in SjS group, $100 \%$ in overlap and $90 \%$ in SSc patients. PTEN protein was expressed in $87.2 \%$ of patients with SjS, $57.9 \%$ of overlap and $100 \%$ of SSC patients and the difference was statistically different $(P=0.023)$. Although ductal epithelial TGF- $\beta 1$ expression was similar between groups $(P=0.345)$, acinar

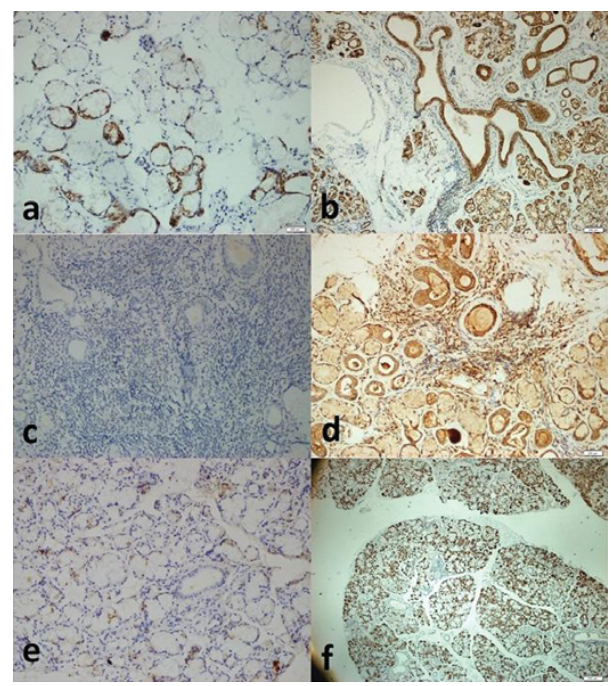

Figure 1. Minor salivary gland biopsy pictures with different stainings of mTOR [1 positive (a) and 3 positive staining (b)], PTEN [negative staining (c) and score 2 (d)] and TGF- $\beta 1$ [negative staining (e) and score $3(\mathrm{f})]$. 
cell expression is more frequent in SSc $(72.7 \%)$ and overlap patients $(85.7 \%)$ in comparison with $\mathrm{SjS}(58.2 \%$ and $P=0.004)$. Additionally, most of the acinar TGF- $\beta 1$ staining was strong positive in SSC patients (45.5\% vs $19.0 \%$ and $3.6 \%$ ). Conclusions: The results of our study showed that mTOR may be one of the common pathways for the pathology/inflammation observed in both SjS and SSc. Thus, there may be a room for mTOR inhibitors for the treatment of both diseases. Additionally PTEN and TGF- $\beta 1$ expression, in particular acinar TGF- $\beta 1$ might be a distinctive feature of SSc.

Disclosure of Interest: None declared

DOI: 10.1136/annrheumdis-2017-eular.5191

\section{THURSDAY, 15 JUNE 2017}

\section{SLE, Sjögren's and APS - clinical aspects (other than treatment)}

\section{THU0246 THE IMPACT OF CLASSIFYING SLE PATIENTS WITH THE SLICC-2012 VERSUS THE ACR-1997 CLASSIFICATION CRITERIA ON EARLY DIAGNOSIS, SEVERITY, AND DAMAGE: DATA FROM THE COMMUNITY-BASED CRETAN LUPUS REGISTRY}

I. Gergianaki ${ }^{1,2}$, A. Fanouriakis ${ }^{3}$, C. Adamichou ${ }^{2}$, G. Spyrou $^{4}$, P. Sidiropoulos ${ }^{2}$, D. Boumpas ${ }^{1,3,5,6,7}, \mathrm{G}$. Bertsias ${ }^{1,2}{ }^{1}$ Laboratory of Autoimmunity and Inflammation, Institute of Molecular Biology-Biotechnology, FORTH:

${ }^{2}$ Rheumatology, Clinical Immunology and Allergy, University of Crete School of Medicine, Iraklio: ${ }^{3} 4$ th Department of Medicine, Attikon University Hospital, National and Kapodistrian University of Athens Medical School, Athens; ${ }^{4}$ Laboratory of Autoimmunity and Inflammation, University of Crete School of Medicine, Iraklio; ${ }^{5}$ Laboratory of Autoimmunity and Inflammation, Biomedical Research Foundation of the Academy of Athens, Athens, Greece; ${ }^{6}$ Medical School, University of Cyprus, Nicosia, Cyprus; ${ }^{7}$ Joint Rheumatology Program, Attikon University Hospital, National and Kapodistrian University of Athens Medical School, Athens, Greece

Background: Systemic Lupus Erythematosus (SLE) often poses challenges to diagnosis in clinical practice and classification in research. The 2012 SLICC criteria have been recently introduced, with validation studies suggesting greater sensitivity yet equal or lower specificity compared to the 1997 ACR criteria. However, the prognostic significance of classifying SLE patients with the SLICC 2012 versus the ACR ${ }^{1997}$ criteria is not known.

Objectives: To evaluate the impact of SLICC ${ }^{2012}$ versus ACR ${ }^{1997}$ SLE classification in terms of clinical characteristics and outcome.

Methods: Both the SLICC 2012 and the ACR ${ }^{1997}$ classification criteria were applied to adult SLE patients enrolled in the community-based Cretan Lupus Registry over the period 1999-2013. Classified cases were assessed at the end of 2013 in terms of disease severity (determined by the severity of manifestations and the use of lupus treatments) and organ damage (assessed by the SLICC/ACR damage index). Cases who fulfilled both criteria during the observation period were categorized according to which set of criteria was satisfied first and then compared for the frequency of individual criteria.

Results: At the end of the observation period (year 2013), fewer SLE patients had been classified with the $\operatorname{SLICC}^{2012}(\mathrm{n}=602)$ as compared with the $\mathrm{ACR}^{1997}(\mathrm{n}=750)$ criteria. The female-to-male ratio (12.6:1 versus $13: 1)$ and the mean $( \pm S D)$ age at the time of diagnosis ( $42 \pm 15$ versus $43 \pm 15$ years) were comparable between SLICC ${ }^{2012}$ and ACR ${ }^{1997}$-classified cases, respectively. Lupus was classified as mild, moderate and severe in $50 \%, 33 \%$ and $17 \%$ of the ACR $^{1997}$ - as compared to $42 \%, 34 \%$ and $23 \%$ of the SLICC 2012 -classified patients $(p<0.001)$. Damage occurred in $30.5 \%$ of the ACR ${ }^{1997}$ versus $36 \%$ of the SLICC ${ }^{2012}$ cohort $(p=0.01)$ despite comparable disease duration. Patients who fulfilled both sets of criteria $(n=512)$ were categorized into three groups based on which criteria were fulfilled first, i.e. group 0 (concurrently: $87 \%$ of cases), group 1 (SLICC ${ }^{2012}$ after ACR ${ }^{1997}$ : $5 \%$ ), and group 2 (SLICC ${ }^{2012}$ before ACR ${ }^{1997}: 8 \%$ ). We found that malar rash and neurologic disorder were significantly more prevalent in group 1 (malar rash: $54.3 \%, 80.8 \%$ and $48.8 \%$ in groups 0,1 and 2 , respectively, $\mathrm{p}=0.02$; neurologic disorder: $3.7 \%, 15.4 \%$ and $0 \%$, respectively, $\mathrm{p}=0.05$ ). Conversely, group 1 patients had significantly lower frequency of non-scarring alopecia $(51.3 \%, 23.1 \%$ and $68.3 \%$, respectively, $\mathrm{p}=0.02)$ and synovitis $(23.9 \%, 0 \%$ and $29.3 \%$, respectively, $\mathrm{p}=0.012$ ).

Conclusions: Application of the SLICC 2012 criteria may result in classification of SLE patients with more severe disease compared to those who fulfill the $\mathrm{ACR}^{1997}$ criteria, which may have important implications in terms of trial design; however, these data need to be validated. Lack of inclusion of malar rash in the SLICC 2012 criteria and of non-scarring alopecia in the ACR ${ }^{1997}$ criteria may delay the classification of SLE patients.

Disclosure of Interest: None declared

DOI: 10.1136/annrheumdis-2017-eular.6734

\section{THU0247 THE PRESENCE OF ANTI-RO AND ANTI-LA ANTIBODIES IS ASSOCIATED WITH TUBULOINTERSTITIAL DAMAGE IN LUPUS NEPHRITIS}

A. Londono Jimenez ${ }^{1}$, W.B. Mowrey ${ }^{2}$, A. Broder ${ }^{3} .{ }^{1}$ Department of Medicine, Montefiore Medical Center/Albert Einstein College of Medicine; ${ }^{2}$ Epidemiology and Population Health, Albert Einstein College of Medicine; ${ }^{3}$ Division of Rheumatology, Montefiore Medical Center/Albert Einstein College of Medicine, Bronx, United States

Background: Moderate-to-severe tubulointerstitial damage (TID) is associated with poor renal outcomes in lupus nephritis (LN) independent of glomerular pathology ${ }^{1}$. Specific antibody profiles associated with TID in LN have not been identified. Unlike glomerular damage, TID is not associated with anti-dsDNA or complement levels ${ }^{1}$. An association between TID and the presence of anti-Ro/La antibodies has been proposed in Sjögren's syndrome ${ }^{2}$. Whether these antibodies are associated with TID in LN is not known.

Objectives: To study an association between anti-Ro/La antibodies and moderateto-severe TID in LN.

Methods: We identified all patients who fulfilled ACR and/or SLICC criteria for SLE. Patients were included if they had an index renal biopsy consistent with LN between January 2005 and July 2015 and had complete data on TID and anti-Ro/La. Medical history, demographic and laboratory data were ascertained from chart review. TID was defined as the presence of moderate or severe tubular atrophy and/or interstitial fibrosis from the renal biopsy reports.

Results: of the $157 \mathrm{LN}$ patients, 39 (25\%) had moderate/severe TID (Table). As expected, moderate/severe TID was associated with older age, class III/IV $\pm \mathrm{V}$ LN and lower estimated glomerular filtration rate (eGFR) at biopsy. Anti-Ro antibodies were present in $55(47 \%)$ of patients with none/mild TID and 17 (44\%) of patients with moderate/severe TID $(\mathrm{p}=0.74)$. Both anti-Ro and anti-La antibodies were present in $11(9 \%)$ of patients with none/mild TID vs $11(28 \%)$ of patients with moderate/severe TID ( $p=0.003)$. In the logistic regression model adjusted for age, eGFR and LN class, the presence of both anti-Ro and anti-La antibodies was associated with a 3 -fold increase in the odds of TID, OR $3.1,95 \% \mathrm{Cl}(1.1-9.1)$, $\mathrm{p}=0.04$.

Baseline characteristics by TID (none/mild vs. moderate/severe)

\begin{tabular}{lccc}
\hline & $\begin{array}{c}\text { None/Mild TID } \\
(\mathrm{n}=118)\end{array}$ & $\begin{array}{c}\text { Moderate/Severe TID } \\
(\mathrm{n}=39)\end{array}$ & $\mathrm{p}$-value \\
\hline Age, median (IQR), years & $26(17,37)$ & $41(25,53)$ & $<0.001$ \\
Men, $\mathrm{n}(\%)$ & $21(18)$ & $10(26)$ & 0.29 \\
Black Race, n (\%) & $55(47)$ & $22(56)$ & 0.29 \\
Hispanic ethnicity, n (\%) & $41(40)$ & $12(38)$ & 0.79 \\
Charlson comorbidity index, median (IQR) & $3(1,4)$ & $3(1,4)$ & 0.09 \\
Creatinine (mg/dL), median (IQR) & $0.8(0.6,1.2)$ & $1.6(1,2.6)$ & $<0.001$ \\
eGFR mL/min/1.73m², median (IQR) & $91(61,127)$ & $42(26,75)$ & $<0.001$ \\
Protein/Creatinine ratio (mg/mg), & & & \\
$\quad$ median (IQR) & $2.2(1.0,4.9)$ & $2.1(1.5,5.5)$ & 0.68 \\
LN class n (\%) & & & 0.008 \\
l/II & $10(9)$ & 0 & \\
III/IV \pm V & $71(61)$ & $34(87)$ & \\
V & $35(30)$ & $5(13)$ & \\
Low C3, n (\%) & $83(75)$ & $23(70)$ & 0.51 \\
Low C4, n (\%) & $76(70)$ & $22(67)$ & 0.69 \\
Elevated dsDNA, n (\%) & $70(68)$ & $21(66)$ & 0.81 \\
Anti-Ro, n (\%) & $55(47)$ & $17(44)$ & 0.74 \\
Anti-Ro and anti-La, n (\%) & $11(9)$ & $11(28)$ & 0.003 \\
\hline
\end{tabular}

Conclusions: The presence of anti-Ro and anti-La antibodies is associated with moderate/severe TID, independent of age, LN class and eGFR. Understanding the role of anti-Ro/La in the mechanisms underlying TID in LN may lead to novel preventive and therapeutic strategies.

References:

[1] Yu F, et al. Kidney Int 2010;77:820-9.

[2] Francois H, et al. Nat Rev Nephrol, 2016;12(2):82-93.

Disclosure of Interest: None declared

DOI: 10.1136/annrheumdis-2017-eular.2237

\section{THU0248 THE ASSOCIATION BETWEEN SYSTEMIC LUPUS ERYTHEMATOSUS TO BIPOLAR DISORDER - A REAL-LIFE STUDY}

S. Tiosano ${ }^{1,2}$, O. Gendelman ${ }^{1,2}$, D. Comaneshter ${ }^{3}$, H. Amital ${ }^{1,2}$, A. Cohen ${ }^{3,4}$ D. Amital ${ }^{5}$. ${ }^{1}$ Sackler School of Medicine, Tel-Aviv University, Tel-Aviv; ${ }^{2}$ Internal Medicine 'B', Sheba Medical Center, Ramat-Gan; ${ }^{3}$ Chief physician's office, Clalit Health Services, Tel-Aviv; ${ }^{4}$ Siaal Research Center for Family Medicine and Primary Care, Faculty of Health Sciences, Ben Gurion University of the Negev, Beer Sheva; ${ }^{5}$ Ness Ziona Beer Yaacov Mental Health Center, Beer Yaacov, Israel

Background: Systemic Lupus Erythematosus (SLE) is a chronic, autoimmune disease that has a wide variety of physical manifestations, including neuropsychiatric features. Bipolar Disorder (BD) is a chronic, phasic affective disorder that may present as depression or as mania. Neuropsychiatric symptoms in SLE develop in $20 \%>70 \%$ of SLE patients during the course of the disease and in half of these patients they precede the diagnosis of $\mathrm{SLE}^{1-4}$. In half of the patients, neuropsychiatric manifestations occur prior to the diagnosis of SLE ${ }^{5}$. 